OPEN ACCESS

Edited by:

Fumiya Kurosaki,

University of Toyama, Japan

Reviewed by:

Frantisek Baluska,

University of Bonn, Germany

Patrick H. Masson,

University of Wisconsin-Madison,

USA

*Correspondence: Haitao Shi

haitaoshi@hainu.edu.cn Hongli Luo

hlluo@hainu.edu.cn Chaozu He

czhe@hainu.edu.cn

†These authors have contributed equally to this work.

Specialty section: This article was submitted to Plant Metabolism

and Chemodiversity,

a section of the journal

Frontiers in Plant Science

Received: 05 September 2016 Accepted: 29 November 2016 Published: 15 December 2016

Citation:

Wang Q, An B, Wei Y, Reiter RJ,

Shi H, Luo H and He C (2016) Melatonin Regulates Root Meristem

by Repressing Auxin Synthesis and Polar Auxin Transport in Arabidopsis.

Front. Plant Sci. 7:1882.

doi: 10.3389/fpls.2016.01882

\section{Melatonin Regulates Root Meristem by Repressing Auxin Synthesis and Polar Auxin Transport in Arabidopsis}

\author{
Qiannan Wang ${ }^{1 \dagger}$, Bang An ${ }^{1+}$, Yunxie Wei', Russel J. Reiter ${ }^{2}$, Haitao Shi ${ }^{1 *}$, Hongli Luo ${ }^{1 *}$ \\ and Chaozu $\mathrm{He}^{1 *}$
}

${ }^{1}$ Hainan Key Laboratory for Sustainable Utilization of Tropical Bioresources, College of Agriculture, Hainan University, Haikou, China, ${ }^{2}$ Department of Cell Systems and Anatomy, The University of Texas Health Science Center, San Antonio, TX, USA

Melatonin ( $N$-acetyl-5-methoxytryptamine) plays important roles in regulating both biotic and abiotic stress tolerance, biological rhythms, plant growth and development. Sharing the same substrate (tryptophan) for the biosynthesis, melatonin and auxin also have similar effects in plant development. However, the specific function of melatonin in modulating plant root growth and the relationship between melatonin and auxin as well as underlying mechanisms are still unclear. In this study, we found high concentration of melatonin remarkably inhibited root growth in Arabidopsis by reducing root meristem size. Further studies showed that melatonin negatively regulated auxin biosynthesis, the expression of PINFORMED (PIN) proteins as well as auxin response in Arabidopsis. Moreover, the root growth of the triple mutant pin1pin3pin7 was more tolerant than that of wild-type in response to melatonin treatment, suggesting the essential role of PIN1/3/7 in melatonin-mediated root growth. Combination treatment of melatonin and 5-Triiodobenzoic acid (TIBA) did not enhance melatonin-mediated reduction of root meristem size, indicating that polar auxin transport (PAT) may be necessary for the regulation of root meristem size by melatonin treatment. Taken together, this study indicates that melatonin regulates root growth in Arabidopsis, through auxin synthesis and polar auxin transport, at least partially.

Keywords: melatonin, auxin, root meristem, auxin synthesis, polar auxin transport, Arabidopsis

\section{INTRODUCTION}

Melatonin ( $N$-acetyl-5-methoxytryptamine), a widely distributed endogenous bio-molecule in mammals, was first discovered in the bovine pineal gland in 1958 (Lerner et al., 1958). Melatonin regulates many important physiological processes in mammals, including sleep, body temperature regulation, circadian rhythms, mood, immune processes, etc. (Jan et al., 2009; Hardeland et al., 2012; Carrillo-Vico et al., 2013; Reiter et al., 2014).

The existence and discovery of melatonin in other species, especially in higher plants, indicates its extensive functions (Poeggeler et al., 1991; Dubbels et al., 1995; Hattori et al., 1995). Numerous studies have shown that melatonin is widely involved in regulating both the biotic and abiotic stress tolerance, biological rhythms, plant growth and development (seed germination, root architecture, shoot development, plant flowering, fruit ripening, and yield;

Abbreviations: IAA, indolyl-3-acetic acid; TIBA, 5-Triiodobenzoic acid. 
Kang et al., 2010; Okazaki et al., 2010; Wang et al., 2012; Byeon et al., 2013, 2014b; Yin et al., 2013; Zhang et al., 2013, 2014; Zhao et al., 2013; Byeon and Back, 2014a; Shi and Chan, 2014; Weeda et al., 2014; Shi et al., 2015a,b,c,d,e, 2016).

In higher plants, melatonin is synthesized from tryptophan as substrate by four key enzymes [tryptophan decarboxylase (TDC), tryptamine 5-hydroxylase (T5H), serotonin $N$-acetyltransferase (SNAT), and $N$-acetylserotonin $O$-methyltransferase (ASMT)] (Kang et al., 2007a,b, 2011, 2013; Okazaki et al., 2009; Fujiwara et al., 2010; Byeon and Back, 2014b, 2015; Zuo et al., 2014; Arnao and Hernández-Ruiz, 2015). Recent studies found that $N$-acetylserotonin can also be synthesized via caffeic acid O-methyltransferase (COMT) in Arabidopsis thaliana, except ASMT (Byeon et al., 2014a; Lee et al., 2014). Interestingly, there are two different pathways for the synthesis of auxin (IAA) in plants, one is tryptophan-independent, and the other is tryptophan-dependent, sharing the same substrate with melatonin (Wang et al., 2015). Tryptophandependent pathway is dependent on precursor tryptophan, through tryptamine (TAM), indole-3-pyruvic acid (IPyA) and indole-3-acetaldoxime (IAOx) pathways (Benjamins and Scheres, 2008; Strader and Bartel, 2008; Chandler, 2009).

Melatonin also showed some similar functions as auxin in the higher plants, in addition to sharing the same substrate for their biosynthesis. Previous studies suggested that melatonin acts as a growth-stimulating molecule in lupin tissues and some monocots, including canary grass, wheat, barely, and oat; its IAA-like activity is $10-55 \%$ of that of auxin (Hernández-Ruiz et al., 2004, 2005). However, there are also reports indicating that melatonin regulates Arabidopsis root growth independent of auxin signaling (Pelagio-Flores et al., 2012; Koyama et al., 2013).

To date, the function of melatonin in regulating root growth and the underlying mechanisms are still unclear in higher plants. Moreover, the relationship between melatonin and auxin remains unknown. In the present work, different concentrations of melatonin were used to treat the wild-type (WT, Col-0) Arabidopsis. The results showed that melatonin significantly suppressed root growth by reducing the size of root meristem. Additionally, comprehensive analyses of auxin synthesis, PIN (PINFORMED) proteins and a auxin response marker line of Arabidopsis (DR5 promoter marker line) suggested that melatonin might regulate the root growth through auxin signaling, at least partially.

\section{MATERIALS AND METHODS}

\section{Plant Materials and Growth Conditions}

The ecotype Columbia-0 (Col-0) of Arabidopsis thaliana was used as the WT plant in this study. Other plant materials are listed as follows: pin1 (Salk_047613), pin3 (CS9364), and pin7 (CS9367) from the Arabidopsis Biological Resource Centre (ABRC), pin3pin7 (Benková et al., 2003), pin1pin3pin7 (Blilou et al., 2005), PIN1::PIN1-GFP (Benková et al., 2003), PIN3::PIN3-GFP (Blilou et al., 2005), PIN7::PIN7-GFP (Blilou et al., 2005), DR5::GUS (Sabatini et al., 1999), and DR5::GFP
(Friml et al., 2003). Arabidopsis seeds were sterilized with 70\% (v/v) ethanol for $1 \mathrm{~min}$ and $1 \%$ sodium hypochlorite for $16 \mathrm{~min}$. After washing with distilled water for 3-5 times, seeds were sown on $1 / 2$ Murashige and Skoog medium with $1 \%$ sucrose and $0.8 \%$ agar. The plates with seeds were placed at $4^{\circ} \mathrm{C}$ for 2 days to break dormancy prior to transfer to a culture room under dark/light cycles of $8 \mathrm{~h} / 16 \mathrm{~h}$ at the temperature of $22^{\circ} \mathrm{C}$. Plates were maintained in a vertical position for 3 days in the culture room before various treatments.

\section{Drug Treatments and Root Assay}

As described above, 3-day-old Arabidopsis seedlings were transferred to $1 / 2$ MS medium containing different concentrations of chemical components [melatonin, 2,3,5Triiodobenzoic acid (TIBA) and IAA] for treatments. To limit the effect of solvent, the same volume of solvent including ethanol was used as a control. Thereafter, photos were taken by a digital camera, and the length of primary roots was determined by software Image $\mathrm{J}^{1}$ (version $1.47 \mathrm{~g}$ ). For the root meristem size measurement, every five roots were cut and transferred onto a glass slide, and were treated with clearing solution (30 mL dd $\mathrm{H}_{2} \mathrm{O}, 53.3 \mathrm{~g}$ chloral hydrate and $10 \mathrm{~mL}$ glycerol) for $5 \mathrm{~min}$ before microscope analyses. Images were captured by Leica DM6000 differential interference contrast microscope. The zone between two white arrows in images include both the apical meristem and the transition zone (Verbelen et al., 2006; Baluska et al., 2010). Root meristem size was quantified as previously described (Liu et al., 2015; Yuan and Huang, 2016). Results presented are average values of more than 30 seedlings per treatment from three independent experiments. Statistical analysis was conducted in KaleidaGraph 4.03 .

\section{GUS Staining}

GUS staining was performed as described previously (Jefferson et al., 1987). Samples were cleared as mentioned above before observation. For DR5::GUS marker line, $2 \mathrm{~h}$ was enough for staining. The images of GUS staining were taken with a Leica DM6000 microscope equipped with Leica Application Suite software.

\section{Confocal Microscopy}

Arabidopsis seedlings expressing PIN1::PIN1-GFP, PIN3::PIN3GFP, PIN7::PIN7-GFP, and DR5::GFP were observed under Leica TCS SP8 laser scanning confocal microscope, with excitation of $488 \mathrm{~nm}$ argon laser, and emission wavelength range of $505-525 \mathrm{~nm}$. The intensity of argon laser in laser configuration and intensity of laser line 488 in acquire section was set to 20 and 15\%, respectively. Pinhole was set to 1.8 Airy units for all materials. To compare the fluorescent intensity of GFP in roots between control and samples treated, all optical sections were acquired under identical conditions. Quantification of the fluorescent intensity was performed by measuring the mean gray value using Image $J$ software. Since PIN1 is mainly localized in the provasculature in roots, and that both PIN3 and PIN7 are expressed in provasculature and root cap. For the

${ }^{1}$ http://rsbweb.nih.gov/ij/ 
PIN1::PIN1-GFP roots, only the signals in the provasculature were quantified, while for PIN3::PIN3-GFP and PIN7::PIN7-GFP roots, signals both in the provasculature and root cap were quantified separately. And we did not distinguish signals at the plasma membrane from signals in the cytoplasm.

\section{Quantitative Real-Time PCR Analysis}

Three-day-old Arabidopsis seedlings were transferred to new $1 / 2 \mathrm{MS}$ medium and medium containing $600 \mu \mathrm{M}$ melatonin. After another 7 days' treatment, root tips (sections from root meristem to the tip) of control and samples were dissected under a dissecting microscope, and total RNA was isolated from root tips treated with TRIzol reagent (Invitrogen). For cDNA synthesis, $2 \mu \mathrm{g}$ of total RNA from different samples was used for reverse transcription with RevertAid First Strand cDNA Synthesis Kit (Thermo Scientific) according to the manufacturer's recommendations. To analyze the transcript levels of auxinrelated genes in control and treated roots, quantitative realtime PCR was performed with Applied Biosystems 7500 (Foster City, CA, USA) in a $20-\mu \mathrm{L}$ reaction volume containing SYBR Green dye (SYBR Premix Ex Taq, TAKARA). PDF2 (protein phosphatase 2, AT1G13320) was chosen as an internal control (Czechowski et al., 2005). Relative expression levels were estimated using the $2^{-\Delta \Delta C t}$ method (Livak and Schmittgen, 2001). All the primers used in the study are listed in Supplementary Table S1.

\section{Determination of Endogenous Melatonin and IAA Levels in Arabidopsis Roots}

For the endogenous melatonin and IAA measurements, 3days-old seedlings were transferred to new control 1/2 MS medium and medium containing IAA or melatonin for another 8 days. Endogenous melatonin in Arabidopsis root tips was extracted as previously described (Pape and Lüning, 2006). The levels of melatonin and IAA in root extracts were quantified using melatonin enzyme linked immunosorbent assay kit (EKDSM; Buhlmann Laboratories AG, Schonenbuch, Switzerland) and Plant IAA enzyme-linked immunosorbent assay (EIASA) Kit (Jianglai Biotechnology, Shanghai, China), respectively, according to the instructions.

\section{RESULTS}

\section{Melatonin Suppressed the Primary Root Growth in Arabidopsis by Reduced Root Meristem}

To investigate the effects of melatonin on primary root growth in Arabidopsis, 3-day-old WT (Col-0) seedlings were transferred to new $1 / 2$ MS media with different concentrations of melatonin for another 6 days (Figure 1). By measuring and statistical analysis, we found that the primary root length was decreased after melatonin treatment, and the inhibition effect of melatonin exhibited dose-dependent (Figure 1). The result suggested that high concentration of melatonin could suppress the primary root growth in Arabidopsis.

In plants, postembryonic root growth is sustained by the root apical meristem (RAM), which consists of stem cell-like cells that are the precursors of all differentiated cell types (Laux and Mayer, 1998; Dinneny and Benfey, 2008). So we wonder if melatonin to reduce the primary root length by affecting root meristem. To test our hypothesis, 3-day-old seedlings were kept growing under different concentrations of melatonin for another 6 days, and we found that both the number of meristem cells and the length of meristem are significantly reduced with increased concentration of melatonin (Figures 2B,C), indicating that melatonin-mediated repression of primary root growth might be due to reduced root meristem.

Our data showed that $10 \mu \mathrm{M}$ melatonin had no effect on primary root growth (Figures 1 and 2), and our previous work suggested that $10-50 \mu \mathrm{M}$ melatonin had litter effect on endogenous melatonin content (Shi et al., 2015c). Therefore, we chose high concentration of melatonin for further analyses in this study.
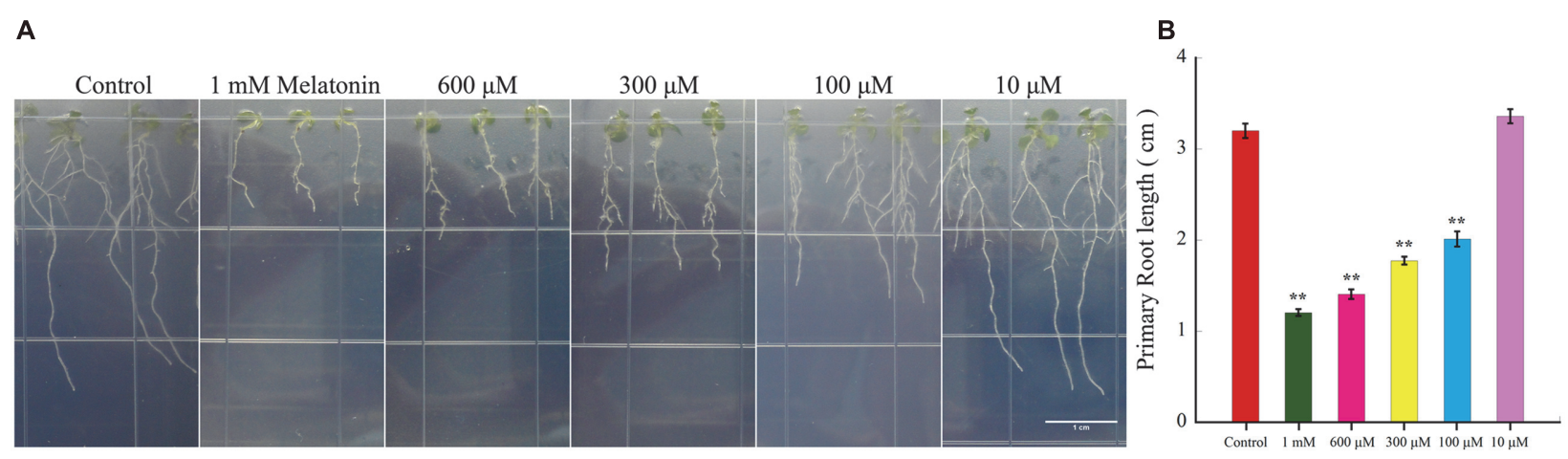

FIGURE 1 | Effect of high concentration of Melatonin on the length of Arabidopsis primary root. After 3 days' culture, seedlings were transferred to $1 / 2$ MS medium with indicated concentrations of melatonin for other 6 days, and the primary root length were measured with software Image J. (A) Digital images of wild-type Arabidopsis seedlings treated with different concentrations of melatonin. Scale bar $=1 \mathrm{~cm}$. (B) Primary root length of Arabidopsis growing on medium with control and increasing concentration of melatonin. More than 25 seedlings per experiment from three independent experiments were measured for statistic analysis. Values represent mean $\pm \mathrm{SD},{ }^{*} P<0.01$ by a Student's $t$-test. 


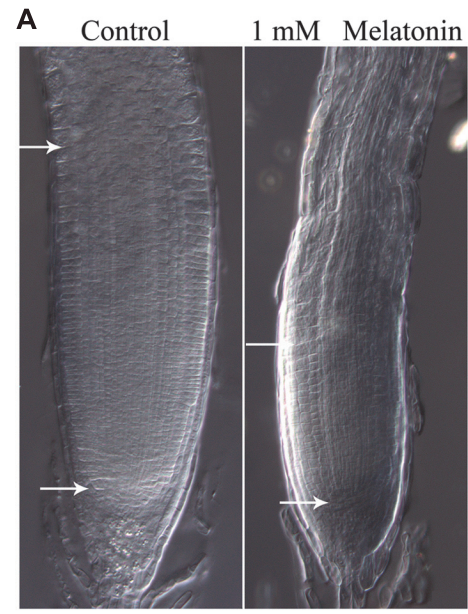

B

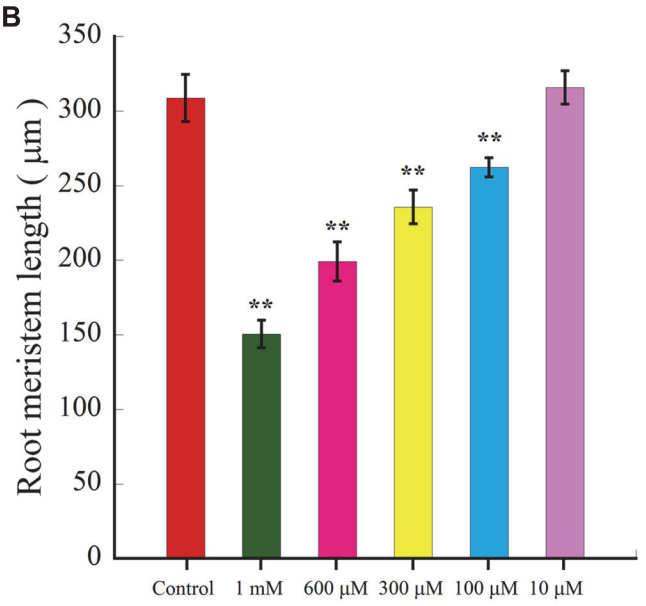

$300 \mu \mathrm{M}$
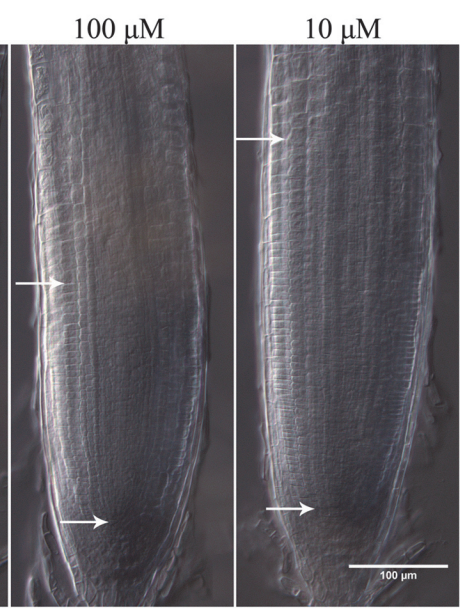

C

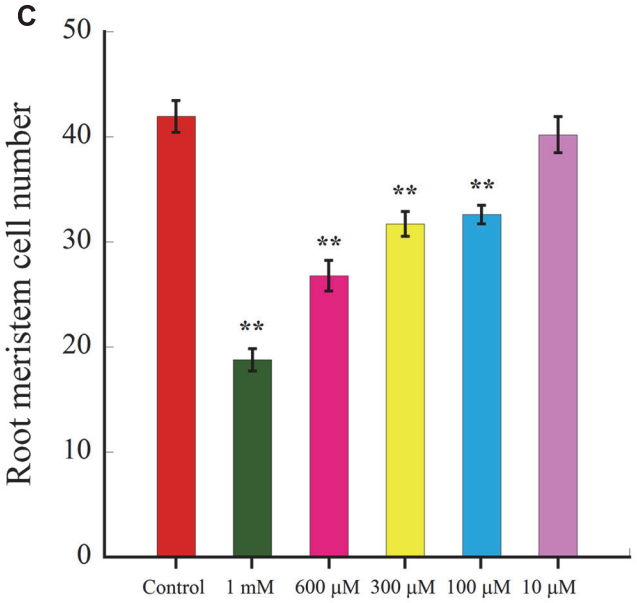

FIGURE 2 | Effects of Melatonin on the size of the Arabidopsis root meristem. Three-day-old seedlings were kept growing under different concentrations of melatonin for other 6 days. More than 25 seedlings per experiment from three independent experiments were cleared for imaging. Values represent mean $\pm \mathrm{SD}$, ${ }^{* *} P<0.01$ by a Student's $t$-test. (A) Images of Arabidopsis root tips treated with melatonin for 6 days were present. Scale bar $=100 \mu \mathrm{m}$. (B) Comparison of root meristem length of the Arabidopsis seedlings treated with different levels of melatonin. (C) Quantification of root meristem cell number of seedlings treated with different levels of melatonin. More than 25 seedlings per experiment from three independent experiments were measured for statistic analysis. Values represent mean $\pm \mathrm{SD},{ }^{* *} P<0.01$ by a Student's $t$-test.

\section{Melatonin Negatively Regulated Auxin Biosynthesis}

Since defective auxin response can cause reduced meristem phenotype, the first question we wanted to known was whether melatonin actually affects auxin biosynthesis. YUCCA (YUC) proteins, TRYPTOPHAN AMINOTRANSFERASE OF ARABIDOPSIS (TAA) family, TAA RELATED 1 and 2 play important roles in auxin (IAA) biosynthesis during plant development (Cheng et al., 2006; Yamamoto et al., 2007; Stepanova et al., 2008; Tao et al., 2008), so we investigated the effects of melatonin on the transcript levels of these genes (Figure 3A). Quantitative real-time PCR showed that the transcript levels of YUC1, YUC2, YUC5, YUC6, and TAR2 significantly decreased after $600 \mu \mathrm{M}$ melatonin treatment. The transcript levels of YUC3, YUC4, YUC7, and YUC8 increased after treatment, while the relative expression levels of YUC3 and YUC8 in roots with treatment were less than 1.5-fold in control.
Indeed, the endogenous IAA content in melatonin-treated roots was significantly lower than that of control (Figure 3B).

\section{Melatonin Repressed Polar Auxin Transport in Arabidopsis}

PINFORMED proteins, especially PIN1, PIN3, and PIN7, directly participate in auxin transport in plant roots (Friml et al., 2003; Blilou et al., 2005), and play important roles in controlling the size of root meristem. In order to examine whether melatonin treatment affected the levels of these proteins in root, we measured the relative fluorescence intensity of GFP using the marker lines PIN1::PIN1-GFP, PIN3::PIN3-GFP, and PIN7::PIN7GFP. As shown in Figure 4, signals of PIN1 in provasculture, and signals of PIN3 and PIN7 deriving from both root cap and provasculture region were decreased significantly after $600 \mu \mathrm{M}$ treatment. The quantitative real-time PCR demonstrated that the relative transcript levels of PIN1, PIN3, and PIN7 were also 


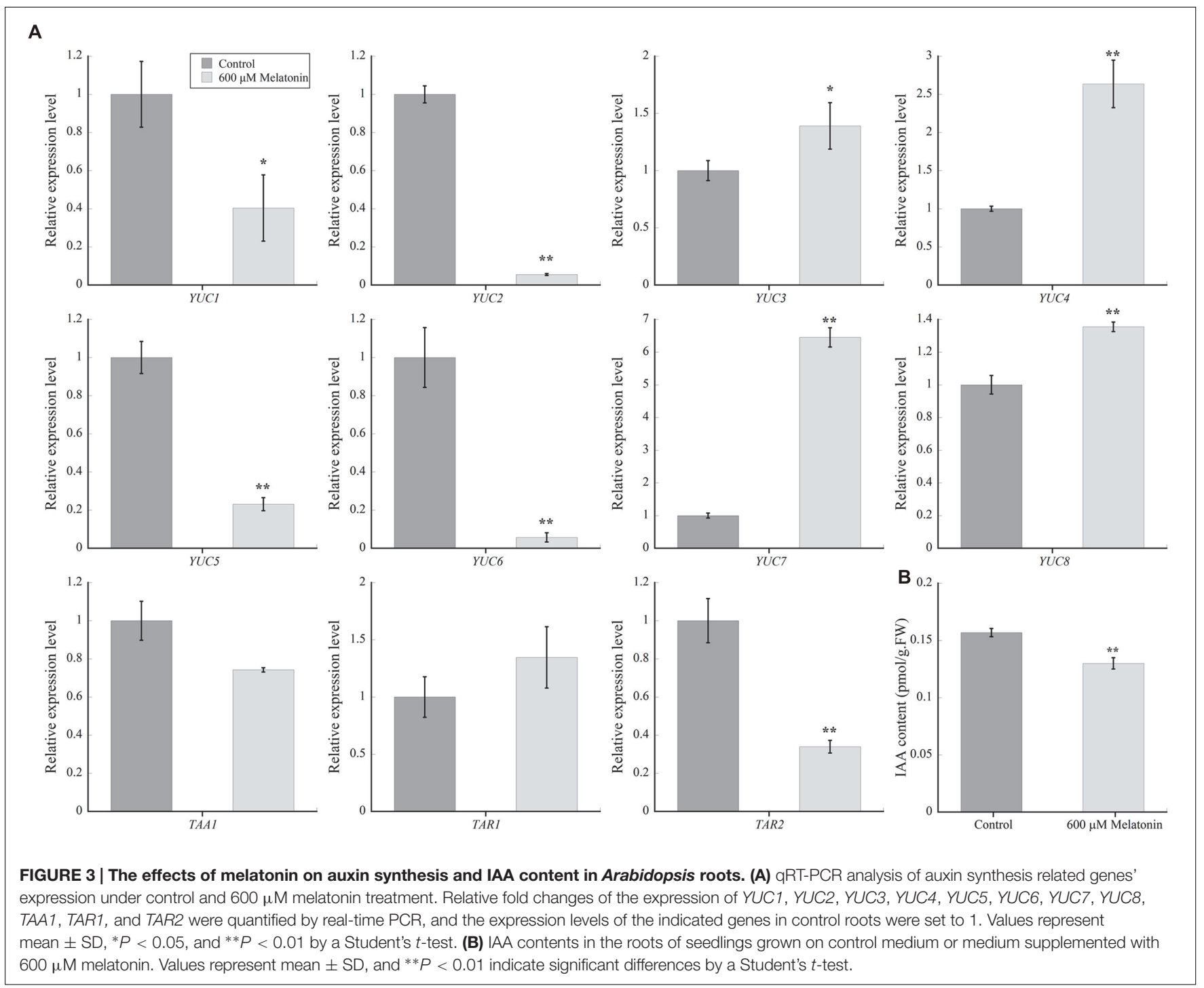

significantly reduced in melatonin-treated roots, suggesting that melatonin treatment repressed the expression of PIN1, PIN3 and PIN7. To further confirm the involvement of PINs in melatoninmediated root development, the meristem length and cell number of the roots of PIN mutants (including pin1, pin3, pin7, pin3pin7, and pin1pin3pin7) were also determined. Notably, we found that the root growth of triple mutant pin1pin3pin7 was more tolerant to melatonin treatment than WT and other mutants (Figures 4D,E), indicating the essential role of PIN1/3/7 in melatonin-mediated repression of root meristem.

\section{Melatonin Repressed Auxin Response in Arabidopsis in an IAA Similar Manner}

Endogenous auxin level is directly related to development of plant roots. To further dissect the underlying mechanism of melatonin during Arabidopsis root growth and the relationship between melatonin and auxin, exogenous IAA and auxin transport inhibitor (TIBA) were used to treat the seedlings.
Firstly, 3-day-old seedlings were treated with melatonin containing medium in the presence or absence of $2 \mu \mathrm{M}$ TIBA for 8 days. The root meristem length and cell number were measured. The results showed that both melatonin and TIBA treated roots reduced root meristem length and cell number, but the inhibition caused by melatonin was not intensified by the presence of TIBA (Figures 5A,B), indicating that polar auxin transport (PAT) might be necessary for the regulation of root meristem size by melatonin treatment.

Since melatonin-treated roots had lower IAA levels (Figure 3B), we wonder whether the root meristem size was reduced by melatonin through decreasing IAA content. If so, the reduction of root meristem size could be complemented, or partially complemented by exogenous IAA at a certain concentration. Two concentrations of IAA (0.5 and $100 \mathrm{nM}$ ) were used to treat the seedlings. We found that treatment of $0.5 \mathrm{nM}$ IAA for 8 days alone did not affect the root meristem size, but $0.5 \mathrm{nM}$ IAA and $600 \mu \mathrm{M}$ melatonin cotreatment significantly reduced the root meristem size, similar 

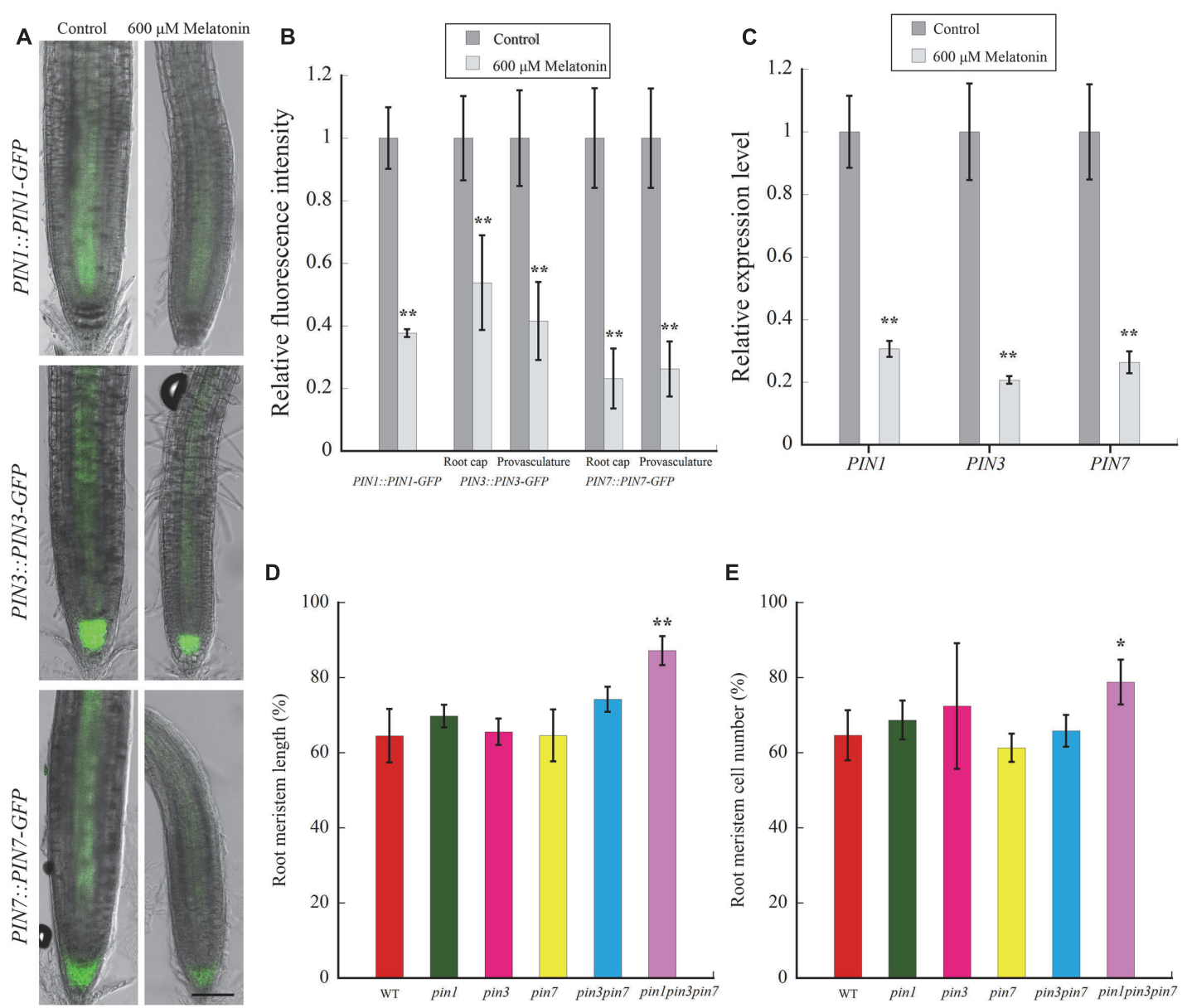

FIGURE 4 | The expression of auxin efflux components PINFORMEDS (PINs) were down-regulated after treatment of $600 \mu M$ melatonin. (A) Effects of Melatonin on the abundance of PIN proteins in Arabidopsis. Three-day-old seedlings harboring indicated markers were transferred to control medium or medium with $600 \mu \mathrm{M}$ melatonin for 6 days. Scale bar = $100 \mu \mathrm{m}$. (B) Comparison of GFP fluorescence intensity in plants treated without or with melatonin as in (A) by Image J. The fluorescence intensity levels of the control roots were set to 1 . Values represent mean $\pm \mathrm{SD}$, ${ }^{* *} P<0.01$ by a Student's $t$-test. (C) qRT-PCR analysis of $P$ IN1, PIN3, and PIN7 in Arabidopsis roots under $600 \mu \mathrm{M}$ melatonin treatment. The expression levels of the indicated genes in control roots were set to 1. Values represent mean $\pm \mathrm{SD},{ }^{* *} P<0.01$ by a Student's $t$-test. (D) Quantification of relative root meristem length of various mutants treated without or with $600 \mu \mathrm{M}$ melatonin for 6 days. Values represent mean $\pm \mathrm{SD},{ }^{* *} P<0.01$ by a Student's $t$-test. (E) Quantification of relative root meristem cell number of various mutants treated without or with $600 \mu \mathrm{M}$ melatonin for 6 days. Values represent mean $\pm \mathrm{SD},{ }^{*} P<0.05$, and ${ }^{* *} P<0.01$ by a Student's $t$-test.

to melatonin-treated roots alone (Figures 5C,D). Application of 100 nM IAA caused reduced root meristem size, as previous reported (Rahman et al., 2007; Strader et al., 2011), but the inhibition of $100 \mathrm{nM}$ was less severe than that of $600 \mu \mathrm{M}$ melatonin. To our surprise, $100 \mathrm{nM}$ IAA and $600 \mu \mathrm{M}$ melatonin co-treatment led to a more serious decrease in root meristem size than that of $600 \mu \mathrm{M}$ melatonin (Figures 5C,D). In the meanwhile, we examined the content of endogenous melatonin in the roots of control and $0.5 \mathrm{nM}$ IAA treated seedlings, and found that $0.5 \mathrm{nM}$ IAA treatment resulted in increased level of melatonin(Figure 5E).

DR5 promoter contains seven tandem repeat sequences of an auxin-responsive element, and it is widely used as a reporter for auxin signaling responses and auxin distribution in Arabidopsis (Ulmasov et al., 1997; Friml et al., 2003). After GUS staining, we found that the distribution of auxin was dramatically changed upon melatonin treatment, as it diffused into lateral root cap (LRC) cells from columella cells (Figure 5F). Similar results could be obtained by the observation of DR5::GFP line seedlings treated with melatonin for 5 days (Figure $5 \mathrm{G}$ ) while $2 \mu \mathrm{M}$ TIBA changed the expression pattern of DR5::GFP in root tips. In combination with melatonin, TIBA-induced auxin signals spread to LRC cells and adjacent meristem cells further (Figure 5G). Exogenous application of $100 \mathrm{nM}$ IAA, but not that of $0.5 \mathrm{nM}$ IAA, caused expansion of auxin signals to the lower part of LRC cells, similar to that of melatonin treatment. Interestingly, in association with melatonin, IAA-induced fluorescence signals spread to the whole distal tips of the roots below QC (Figure 5G), just like seedlings treated with higher concentrations of IAA as reported before (Ottenschläger et al., 2003), suggesting that melatonin aggravated the accumulation of auxin signals in the whole distal tips of roots, in an IAA similar manner. 


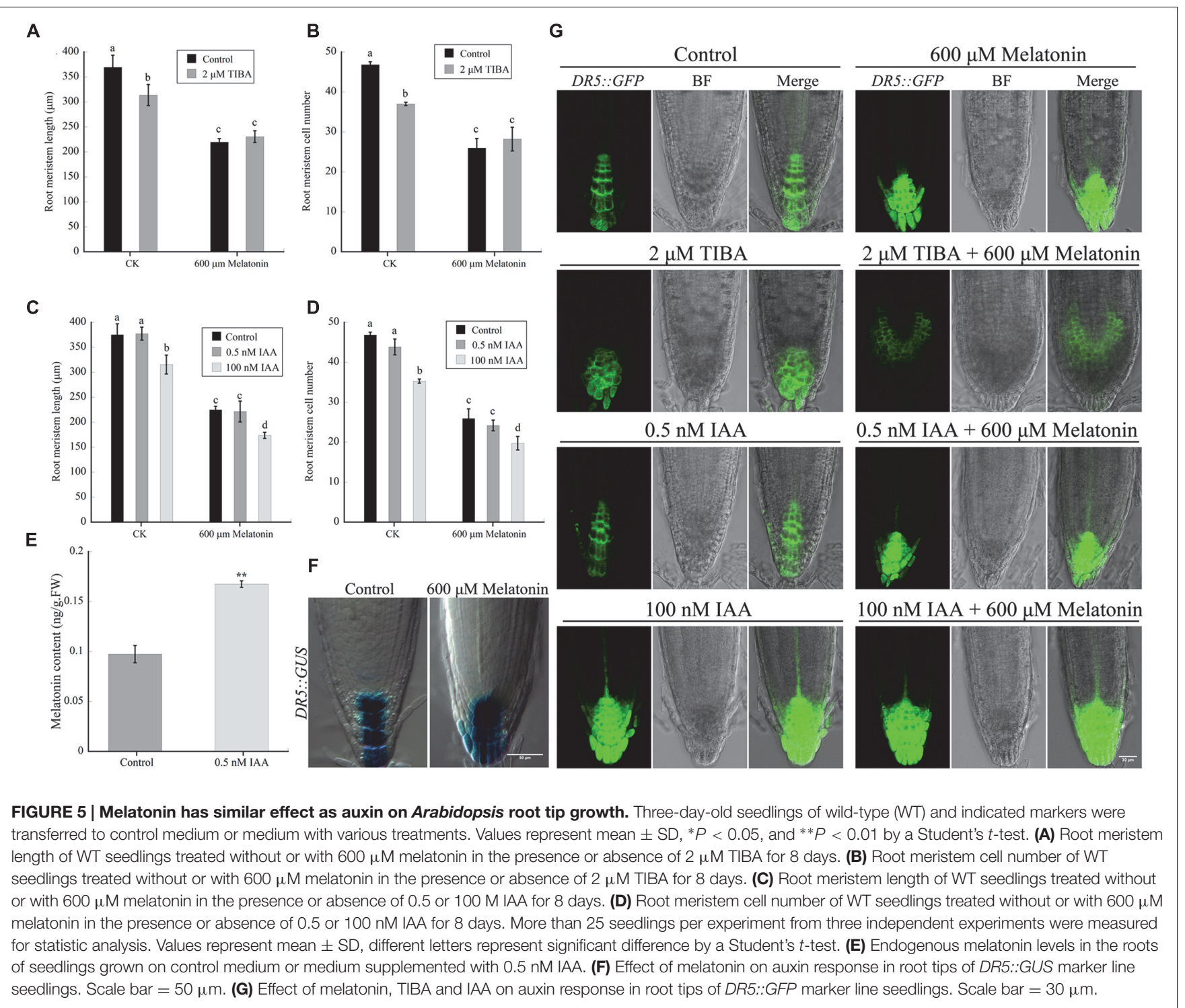

\section{DISCUSSION}

As an important plant hormone, auxin plays vital roles in root cell division, differentiation, elongation, and the overall growth of roots (Benjamins and Scheres, 2008). In recent decades, more attention has been paid to the role of melatonin as a growth regulator of plants (Arnao and Hernández-Ruiz, 2015). Both of auxin and melatonin have been suggested to regulate similar growth processes. Our data showed $10 \mu \mathrm{M}$ melatonin had no effect on primary root growth (Figures 1 and 2), and our previously work suggested that $10-50 \mu \mathrm{M}$ melatonin had litter effect on endogenous melatonin content (Shi et al., 2015c). Based on previous studies and our preliminary experiments, $10-50 \mu \mathrm{M}$ melatonin had litter effect on endogenous melatonin content (Shi et al., 2015c), and lower melatonin concentration also had litter effect on plant root development (Bajwa et al., 2014). Moreover, 100-600 $\mu \mathrm{M}$ melatonin were also widely used in other studies (PelagioFlores et al., 2012; Bajwa et al., 2014). In our study, we first tested the effect of melatonin on root growth of Arabidopsis and found that $100 \mu \mathrm{M}$ melatonin had already shown an inhibitory effect on root growth, in accordance with previous reports (Chen et al., 2009). However, Bajwa et al. (2014) showed that 100,200 , and $400 \mu \mathrm{M}$ melatonin treatment had no significant effects on plant root growth. The difference might be attributed to the big values of SD of their results and solvent effect. To limit the effect of solvent, the same volume of solvent including ethanol was used as a control in this study. Moreover, the average values of more than 30 seedlings per treatment from three independent experiments. The higher the concentration of melatonin, the more suppression of the root length (Figure 1B), indicating that melatonin inhibit root length in a dose-dependent manner. Although moderate auxin promotes root growth of plants, overproduction of auxin 


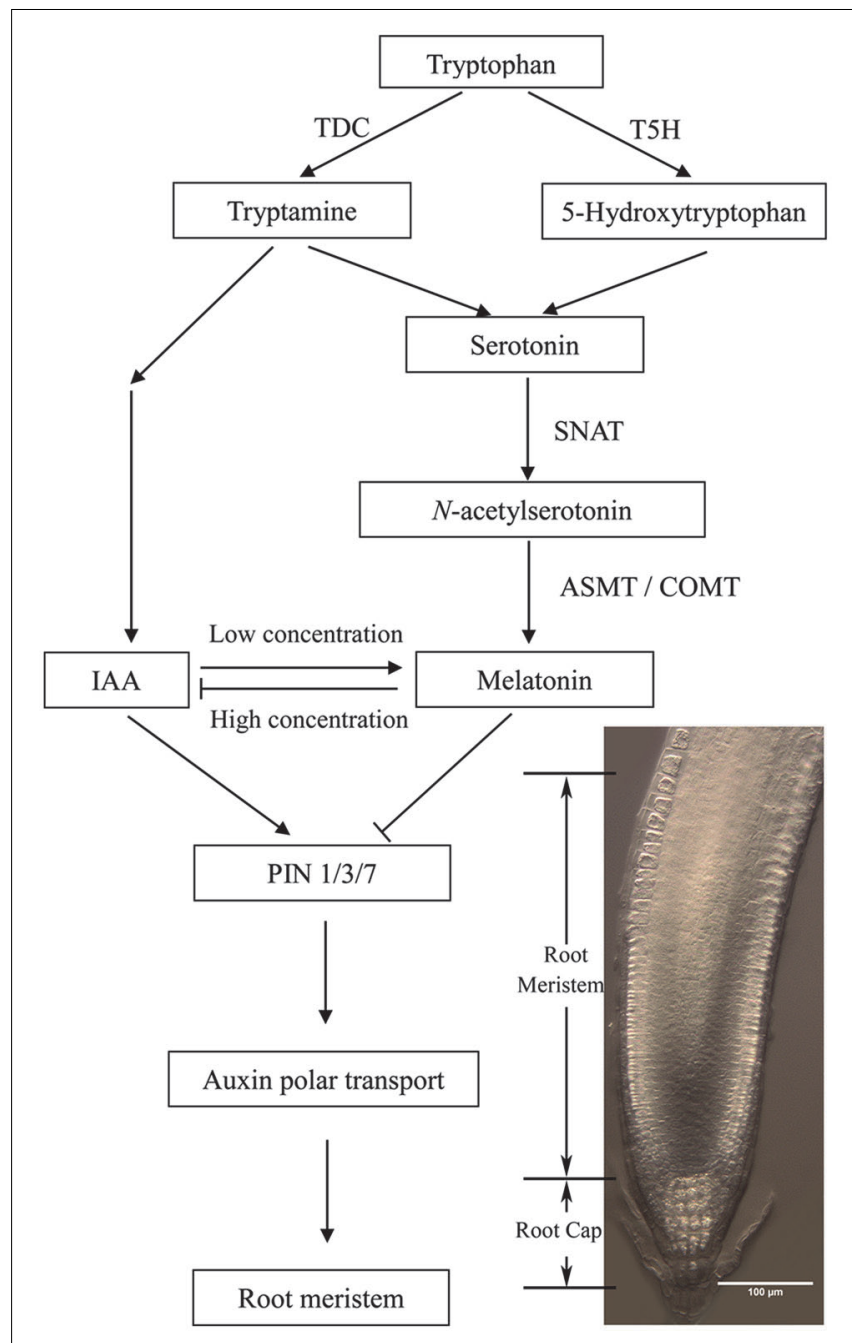

FIGURE 6 | The possible model showing the relationship between melatonin and auxin during plant root growth.

levels can cause a decay of root growth (Teale et al., 2005; Strader et al., 2011). After digging deeper into the effects of melatonin, we found high concentrations of melatonin reduced root meristem size, consistent with its effects on primary root length (Figures 2B,C).

The effects of auxin on root growth, is largely dependent on its biosynthesis and polar transport, which cause optimal auxin accumulation and distribution in the root apex during the whole developmental process (Blilou et al., 2005; Kim et al., 2007; Li et al., 2011). Quantitative real-time PCR showed that the expression levels of YUC1, YUC2, YUC5, YUC6, and TAR2, key genes of auxin biosynthesis, were significantly down-regulated after $600 \mu \mathrm{M}$ melatonin treatment consistent with lower IAA content in $600 \mu \mathrm{M}$ melatonintreated roots (Figure 3). If we set the transcript level of $Y U C 1$ in control material as 1 , the relative expression level of YUC2, YUC3, YUC4, YUC5, YUC6, YUC7, YUC8, TAA1, TAR1, and TAR2 was 28.7, 406.7, 4.6, 0.37, 151.9, 20.6, 170.3,
43.5, 41, and 693 separately. In melatonin-treated material, the relative transcript level of YUC1 and other genes were $0.4,1.6,564.1,11.9,0.1,1,132.8,230.8,23.1,29.6$, and 234.3, respectively. After melatonin treatment, the total relative expression abundance was decreased significantly. The decrease in expression of YUC1, YUC2, YUC5, YUC6, and TAR2, together with the effects of melatonin on auxin transport may cause the decrease in IAA levels in roots, at least partially. As reported recently, application of 1-naphthaleneacetic acid (NAA) and 2,4-dichlorophenoxyacetic acid (2,4-D) results in a decay in the transcript levels of YUC1, YUC2, YUC4, YUC6, and TAR2 in Arabidopsis seedlings (Suzuki et al., 2015). We noticed there is a difference in the endogenous IAA level between this study and previous results (Zhao et al., 2001; Lee et al., 2012), which could be resulted from two possible reasons. One reason could be the sample differences. In our work, only the root tips were harvested and used for analysis. Another possibility is that different methods were used. In this study, we used ELISA method. Based on the consistence between the transcript levels of auxin biosynthesis genes and IAA level, we concluded that melatonin negatively regulated auxin biosynthesis.

Polar auxin transport is essential for the distribution of auxin in Arabidopsis root tips, and the auxin efflux machinery PIN proteins play important roles in controlling the size of root meristem (Friml et al., 2003; Blilou et al., 2005). Expression levels of PINs were always found down-regulated in the shortened root meristem after stresses (Liu et al., 2015; Yuan and Huang, 2016). Even in PIN1::GFP line roots exogenously treated with IAA, a significant decrease in GFP fluorescence was detected under high concentrations (5 and $10 \mu \mathrm{M}$; Omelyanchuk et al., 2016). Coincidently, our results demonstrated a decrease in both of the transcript levels and the protein expression levels of PIN1, PIN3, and PIN7 in melatonin-treated roots (Figures 4A,C, revised Figure 4B). Auxin transport inhibitor TIBA could also decrease the root meristem size and root growth (Blilou et al., 2005). Our study showed auxin transport inhibitor TIBA did not enhance melatonin-mediated reduction of root meristem size, indicating that PAT might be necessary for the regulation of root meristem size by melatonin treatment (Figures 5A,B). Moreover, the triple mutant pin1pin3pin7 was more tolerant than WT in response to melatonin treatment, suggesting that PIN1/3/7mediated PAT might contribute to melatonin-regulated root meristem.

Previous study showed application of low concentration of melatonin $(0.1 \mu \mathrm{M})$, increased the endogenous levels of IAA in Arabidopsis roots (Chen et al., 2009), and our findings demonstrated that exogenous application of low concentration of IAA $(0.5 \mathrm{nM})$, also raised the endogenous melatonin content in roots (Figure 5E). On the contrary, $100 \mathrm{nM}$ IAA caused reduced root meristem size, as previously reported (Rahman et al., 2007; Strader et al., 2011). In this study, simultaneous presence of $100 \mathrm{nM}$ IAA and $600 \mu \mathrm{M}$ melatonin led to more serious decrease in root meristem size than that of $600 \mu \mathrm{M}$ melatonin alone (Figures 5C,D).

The expression of DR5 promoter marker line in root tips represents the responses and distribution pattern of auxin. 
Unlike the results showed by Pelagio and Koyama (PelagioFlores et al., 2012; Koyama et al., 2013), here we found that melatonin was able to change the expression pattern in both of DR5::GUS and DR5::GFP line roots (Figure 5G), just like the effects of IAA (Ottenschläger et al., 2003). In combination with $600 \mu \mathrm{M}$ melatonin, $100 \mathrm{nM}$ IAA caused a more expansion pattern of fluorescence signals in the whole root caps, including columella cells and lateral root cells, indicating that exogenous application of melatonin intensified the effect of IAA on the auxin responses in root tips. Considering altered auxin synthesis in root tip of treated seedlings, decreased signals of PIN1 in provasculature, reduced PIN3 and PIN7 signals in both root cap and provasculature, the DR5::GFP signal should not expand that strongly toward the lateral cap and tip cells after melatonin treatment. Then we also observed the expression and localization of PIN2 in the root cells, however, no significant difference was shown about the expression of PIN2 in cell membrane without and with $600 \mu \mathrm{M}$ melatonin treatment (revised Supplementary Figures S1A-C). What's surprised is that PIN2 signals in cytoplasm in melatonin-treated roots were obviously increased, although the signals in cytoplasm were much lower than that in cell membrane. This may, at least partially explain that why DR5 signals spread into the whole root cap after melatonin treatment. These results indicated that melatonin may have dual and complex effects on auxin transport. Besides PIN1/2/3/7, there may be other issues contribute to melatonin-mediated auxin signaling, which need to be further investigated.

Based on our results, we proposed a working model for the mechanisms by which melatonin regulates root meristem (Figure 6). Melatonin and auxin share the same substrate tryptophan during biosynthetic pathways, and exogenous application of IAA at low concentration increases melatonin production, while high concentration of melatonin decreases the level of IAA and PIN1, 3, 7 in Arabidopsis roots. Thus, melatonin regulates root meristem by repressing auxin synthesis and polar auxin transport in Arabidopsis. In summary, this study provides a direct link between melatonin and root growth, and indicates the novel involvement of auxin responses in melatonin-mediated root growth in Arabidopsis. We highlight the relationship between of melatonin and auxin in Arabidopsis root growth.

\section{REFERENCES}

Arnao, M. B., and Hernández-Ruiz, J. (2015). Functions of melatonin in plants: a review. J. Pineal Res. 59, 1-23. doi: 10.1111/jpi.12253

Bajwa, V. S., Shukla, M. R., Sherf, S. M., Murch, S. J., and Saxena, P. K. (2014). Role of melatonin in alleviating cold stress in Arabidopsis thaliana. J. Pineal Res. 56, 238-245. doi: 10.1111/jpi.12115

Baluska, F., Mancuso, S., Volkmann, D., and Barlow, P. W. (2010). Root apex transition zone: a signalling-response nexus in the root. Trends Plant Sci. 15, 402-408. doi: 10.1016/j.tplants.2010.04.007

Benjamins, R., and Scheres, B. (2008). Auxin: the looping star in plant development. Ann. Rev. Plant Biol. 59, 443-465. doi: 10.1146/annurev.arplant. 58.032806.103805

Benková, E., Michniewicz, M., Sauer, M., Teichmann, T., Seifertová, D., Jürgens, G., et al. (2003). Local, efflux-dependent auxin gradients as a common module

\section{AUTHOR CONTRIBUTIONS}

HS conceived and directed this study, revised the manuscript; QW designed and performed the experiments, analyzed the data, wrote and revised the manuscript; BA performed the experiments, analyzed the data and revised the manuscript; YW provided help in the melatonin and IAA content analysis; RR provided suggestions and revised the manuscript; HL designed the experiments and revised the manuscript; $\mathrm{CH}$ designed the experiments and revised the manuscript. All authors approved the manuscript and the version to be published.

\section{FUNDING}

This work was supported by the Hainan Natural Science Foundation (No. 20163052), the startup funding and the scientific research foundation of Hainan University (No. kyqd1516, No. kyqd1531), and the National Natural Science Foundation of China (No. 31570249).

\section{ACKNOWLEDGMENT}

We thank ABRC and many researchers for sharing their published materials.

\section{SUPPLEMENTARY MATERIAL}

The Supplementary Material for this article can be found online at: http://journal.frontiersin.org/article/10.3389/fpls.2016.01882/ full\#supplementary-material

FIGURE S1 | The expression of PIN2 in cytoplasm of root cells was altered after $600 \mu \mathrm{M}$ melatonin treatment. Three-day-old seedlings harboring indicated markers were transferred to control medium or medium with $600 \mu \mathrm{M}$ melatonin for 6 days. (A) Effects of Melatonin on the expression pattern of PIN2 in Arabidopsis. Scale bar $=50 \mu \mathrm{m}$. (B) Localization of PIN2 in root cells in control seedling and seedling treated with $600 \mu \mathrm{M}$ melatonin. Scale bar $=5 \mu \mathrm{m}$. (C) Comparison of GFP fluorescence intensity in plants treated without or with melatonin as in (A) by Image J. The fluorescence intensity levels of the control roots were set to 1 . Values represent mean $\pm \mathrm{SD},{ }^{*} P<0.05$, and ${ }^{* *} P<0.01$ by a Student's t-test.

for plant organ formation. Cell 115, 591-602. doi: 10.1016/S0092-8674(03) 00924-3

Blilou, I., Xu, J., Wildwater, M., Willemsen, V., Paponov, I., Friml, J., et al. (2005). The PIN auxin efflux facilitator network controls growth and patterning in Arabidopsis roots. Nature 433, 39-44. doi: 10.1038/nature03184

Byeon, Y., and Back, K. (2014a). An increase in melatonin in transgenic rice causes pleiotropic phenotypes, including enhanced seedling growth, delayed flowering, and low grain yield. J. Pineal Res. 56, 408-414. doi: 10.1111/jpi.12129

Byeon, Y., and Back, K. (2014b). Melatonin synthesis in rice seedlings in vivo is enhanced at high temperatures and under dark conditions due to increased serotonin N-acetyltransferase and Nacetylserotonin methyltransferase activities. J. Pineal Res. 56, 189-195. doi: 10.1111/jpi.12111

Byeon, Y., and Back, K. (2015). Molecular cloning of melatonin2-hydroxylase responsible for 2-hydroxymelatonin production in rice (Oryza sativa). J. Pineal Res. 58, 343-351. doi: 10.1111/jpi.12220 
Byeon, Y., Lee, H. Y., Lee, K., and Back, K. (2014a). Caffeic acid O-methyltransferase is involved in the synthesis of melatonin by methylating $\mathrm{N}$-acetylserotonin in Arabidopsis. J. Pineal Res. 57, 219-227. doi: $10.1111 /$ jpi.12160

Byeon, Y., Park, S., Kim, Y. S., and Back, K. (2013). Microarray analysis of genes differentially expressed in melatonin-rich transgenic rice expressing a sheep serotonin N-acetyltransferase. J. Pineal Res. 55, 357-363. doi: 10.1111/jpi.12077

Byeon, Y., Park, S., Lee, H. Y., Kim, Y. S., and Back, K. (2014b). Elevated production of melatonin in transgenic rice seeds expressing rice tryptophan decarboxylase. J. Pineal Res. 56, 275-282. doi: 10.1111/jpi.12120

Carrillo-Vico, A., Lardone, P. J., Álvarez-Sánchez, N., Rodríguez-Rodríguez, A., and Guerrero, J. M. (2013). Melatonin: buffering the immune system. Int. J. Mol. Sci. 14, 8638-8683. doi: 10.3390/ijms14048638

Chandler, J. W. (2009). Local auxin production: a small contribution to a big field. Bioessays 31, 60-70. doi: 10.1002/bies.080146

Chen, Q., Qi, W. B., Reiter, R. J., Wei, W., and Wang, B. M. (2009). Exogenously applied melatonin stimulates root growth and raises endogenous IAA in roots of etiolated seedling of Brassica juncea. J. Plant Physiol. 166, 324-328. doi: 10.1016/j.jplph.2008.06.002

Cheng, Y., Dai, X., and Zhao, Y. (2006). Auxin biosynthesis by the YUCCA flavin monooxygenases controls the formation of floral organs and vascular tissues in Arabidopsis. Genes Dev. 20, 1790-1799. doi: 10.1101/gad.1415106

Czechowski, T., Stitt, M., Altmann, T., Udvardi, M. K., and Scheible, W. R. (2005). Genome-wide identification and testing of superior reference genes for transcript normalization in Arabidopsis. Plant Physiol. 139, 5-17. doi: 10.1104/ pp.105.063743

Dinneny, J. R., and Benfey, P. N. (2008). Plant stem cell niches: standing the test of time. Cell 132, 553-557. doi: 10.1016/j.cell.2008.02.001

Dubbels, R., Reiter, R. J., Klenke, E., Goebel, A., Schnakenberg, E., Ehlers, C., et al. (1995). Melatonin in edible plants identified by radioimmunoassay and by high performance liquid chromatography-mass spectrometry. J. Pineal Res. 18, 28-31. doi: 10.1111/j.1600-079X.1995.tb00136.x

Friml, J., Vieten, A., Sauer, M., Weijers, D., Schwarz, H., Hamann, T., et al. (2003). Efflux-dependent auxin gradients establish the apical-basal axis of Arabidopsis. Nature 426, 147-153. doi: 10.1038/nature02085

Fujiwara, T., Maisonneuve, S., Isshiki, M., Mizutani, M., Chen, L., Wong, H. L., et al. (2010). Sekiguchi lesion gene encodes a cytochrome P450 monooxygenase that catalyzes conversion of tryptamine to serotonin in rice. J. Biol. Chem. 285, 11308-11313. doi: 10.1074/jbc.M109.091371

Hardeland, R., Madrid, J. A., Tan, D. X., and Reiter, R. J. (2012). Melatonin, the circadian multioscillator system and health: the need for detailed analysis of peripheral melatonin signal. J. Pineal Res. 52, 139-166. doi: 10.1111/j.1600079X.2011.00934.x

Hattori, A., Migitaka, H., Iigo, M., Itoh, M., Yamamoto, K., Ohtani-Kaneko, R., et al. (1995). Identification of melatonin in plants and its effects on plasma melatonin levels and binding to melatonin receptors in vertebrates. Biochem. Mol. Biol. Int. 35, 627-634.

Hernández-Ruiz, J., Cano, A., and Arnao, M. B. (2004). Melatonin: growthstimulating compound present in lupin tissues. Planta 220, 140-144. doi: 10. 1007/s00425-004-1317-3

Hernández-Ruiz, J., Cano, A., and Arnao, M. B. (2005). Melatonin acts as a growthstimulating compound in some monocot species. J. Pineal Res. 39, 137-142. doi: 10.1111/j.1600-079X.2005.00226.x

Jan, J. E., Reiter, R. J., Wasdell, M. B., and Bax, M. (2009). The role of the thalamus in sleep, pineal melatonin production, and circadian rhythm sleep disorders. J. Pineal Res. 46, 1-7. doi: 10.1111/j.1600-079X.2008.00628.x

Jefferson, R. A., Kavanagh, T. A., and Bevan, M. W. (1987). GUS fusions: betaglucuronidase as a sensitive and versatile gene fusion marker in higher plants. EMBO J. 6, 3901-3907.

Kang, K., Kong, K., Park, S., Natsagdorj, U., Kim, Y. S., and Back, K. (2011). Molecular cloning of a plant $\mathrm{N}$-acetylserotonin methyltransferase and its expression characteristics in rice. J. Pineal Res. 50, 304-309. doi: 10.1111/j.1600079X.2010.00841.x

Kang, K., Lee, K., Park, S., Byeon, Y., and Back, K. (2013). Molecular cloning of rice serotonin $\mathrm{N}$-acetyltransferase, the penultimate gene in plant melatonin biosynthesis. J. Pineal Res. 55, 7-13. doi: 10.1111/jpi.12011

Kang, K., Lee, K., Park, S., Kim, Y. S., and Back, K. (2010). Enhanced production of melatonin by ectopic overexpression of human serotonin $\mathrm{N}$-acetyltransferase plays a role in cold resistance in transgenic rice seedlings. J. Pineal Res. 49, 176-182. doi: 10.1111/j.1600-079X.2010.00783.x

Kang, S., Kang, K., Lee, K., and Back, K. (2007a). Characterization of tryptamine 5-hydroxylase and serotonin synthesis in rice plants. Plant Cell Rep. 26, 2009-2015. doi: 10.1007/s00299-007-0405-9

Kang, S., Kang, K., Lee, K., and Back, K. (2007b). Characterization of rice tryptophan decarboxylases and their direct involvement in serotonin biosynthesis in transgenic rice. Planta 227, 263-272. doi: 10.1007/s00425-0070614-z

Kim, J. I., Sharkhuu, A., Jin, J. B., Li, P., Jeong, J. C., Baek, D., et al. (2007). yucca6, a dominant mutation in Arabidopsis, affects auxin accumulation and auxin-related phenotypes. Plant Physiol. 145, 722-735. doi: 10.1104/pp.107. 104935

Koyama, F. C., Carvalho, T. L., Alves, E., da Silva, H. B., Azevedo, M. F., Hemerly, A. S., et al. (2013). The structurally related auxin and melatonin tryptophanderivatives and their roles in Arabidopsis thaliana and in the human malaria parasite Plasmodium falciparum. J. Euk. Microbiol. 60, 646-651. doi: 10.1111/ jeu. 12080

Laux, T., and Mayer, K. F. (1998). Cell fate regulation in the shoot meristem. Semin. Cell Dev. Biol. 9, 195-200. doi: 10.1006/scdb.1997.0208

Lee, H. Y., Byeon, Y., Lee, K., Lee, H. J., and Back, K. (2014). Cloning of Arabidopsis serotonin $\mathrm{N}$-acetyltransferase and its role with caffeic acid O-methyltransferase in the biosynthesis of melatonin in vitro despite their different subcellular localization. J. Pineal Res. 75, 418-426. doi: 10.1111/jpi.12181

Lee, M., Jung, J. H., Han, D. Y., Seo, P. J., Park, W. J., and Park, C. M. (2012). Activation of a flavin monooxygenase gene YUCCA7 enhances drought resistance in Arabidopsis. Planta 235, 923-938. doi: 10.1007/s00425-011-1552-3

Lerner, A. B., Case, J. D., Takahashi, Y., Lee, T. H., and Mori, W. (1958). Isolation of melatonin, a pineal factor that lightens melanocytes. J. Am. Chem. Soc. 80, 2587. doi: $10.1021 / \mathrm{ja} 01543 \mathrm{a} 060$

Li, B., Li, Q., Su, Y., Chen, H. A. O., Xiong, L., Mi, G., et al. (2011). Shoot supplied ammonium targets the root auxin influx carrier AUX1 and inhibits lateral root emergence in Arabidopsis. Plant Cell Environ. 34, 933-946. doi: 10.1111/j.13653040.2011.02295.x

Liu, W., Li, R. J., Han, T. T., Cai, W., Fu, Z. W., and Lu, Y. T. (2015). Salt stress reduces root meristem size by nitric oxide-mediated modulation of auxin accumulation and signaling in Arabidopsis. Plant Physiol. 168, 343-356. doi: 10.1104/pp.15.00030

Livak, K. J., and Schmittgen, T. D. (2001). Analysis of relative gene expression data using real-time quantitative PCR and the 2- $\Delta \Delta \mathrm{CT}$ method. Methods 25, 402-408. doi: 10.1006/meth.2001.1262

Okazaki, M., Higuchi, K., Aouini, A., and Ezura, H. (2010). Lowering intercellular melatonin by transgenic analysis of indoleamine 2, 3-dioxygenase from rice in tomato plants. J. Pineal Res. 49, 239-247. doi: 10.1111/j.1600-079X.2010. 00788.x

Okazaki, M., Higuchi, K., Hanawa, Y., Shiraiwa, Y., and Ezura, H. (2009). Cloning and characterization of a Chlamydomonas reinhardtii cDNA arylalkylamine $\mathrm{N}$-acetyltransferase and its use in the genetic engineering of melatonin content in the Micro-Tom tomato. J. Pineal Res. 46, 373-382. doi: 10.1111/j.1600-079X. 2009.00673.x

Omelyanchuk, N. A., Kovrizhnykh, V. V., Oshchepkova, E. A., Pasternak, T., Palme, K., and Mironova, V. V. (2016). A detailed expression map of the PIN1 auxin transporter in Arabidopsis thaliana root. BMC Plant Biol. 16(Suppl. 1):5. doi: 10.1186/s12870-015-0685-0

Ottenschläger, I., Wolff, P., Wolverton, C., Bhalerao, R. P., Sandberg, G., Ishikawa, H., et al. (2003). Gravity-regulated differential auxin transport from columella to lateral root cap cells. PNAS 100, 2987-2991. doi: 10.1073/pnas. 0437936100

Pape, C., and Lüning, K. (2006). Quantification of melatonin in phototrophic organisms. J. Pineal Res. 41, 157-165. doi: 10.1111/j.1600-079X.2006. 00348.x

Pelagio-Flores, R., Muñoz-Parra, E., Ortiz-Castro, R., and López-Bucio, J. (2012). Melatonin regulates Arabidopsis root system architecture likely acting independently of auxin signaling. J. Pineal Res. 53, 279-288. doi: 10.1111/j. 1600-079X.2012.00996.x

Poeggeler, B., Balzer, I., Hardeland, R., and Lerchl, A. (1991). Pineal hormone melatonin oscillates also in dinoflagellates? Naturwissenschaften 78, 268-269. doi: $10.1007 / \mathrm{BF} 01134354$ 
Rahman, A., Bannigan, A., Sulaman, W., Pechter, P., Blancaflor, E. B., and Baskin, T. I. (2007). Auxin, actin and growth of the Arabidopsis thaliana primary root. Plant J. 50, 514-528. doi: 10.1111/j.1365-313X.2007.03068.x

Reiter, R. J., Tan, D. X., and Galano, A. (2014). Melatonin: exceeding expectations. Physiology (Bethesda) 29, 325-333. doi: 10.1152/physiol.00011.2014

Sabatini, S., Beis, D., Wolkenfelt, H., Murfett, J., Guilfoyle, T., Malamy, J., et al. (1999). An auxin-dependent distal organizer of pattern and polarity in the Arabidopsis root. Cell 99, 463-472. doi: 10.1016/S0092-8674(00)81535-4

Shi, H., and Chan, Z. (2014). The cysteine2/histidine2-type transcription factor ZINC FINGER OF ARABIDOPSIS THALIANA 6- activated C-REPEATBINDING FACTOR pathway is essential for melatonin-mediated freezing stress resistance in Arabidopsis. J. Pineal Res. 57, 185-191. doi: 10.1111/jpi.12155

Shi, H., Chen, Y., Tan, D. X., Reiter, R. J., Chan, Z., and He, C. (2015a). Melatonin induces nitric oxide and the potential mechanisms relate to innate immunity against bacterial pathogen infection in Arabidopsis. J. Pineal Res. 59, 102-108. doi: $10.1111 /$ jpi.12244

Shi, H., Jiang, C., Ye, T., Tan, D. X., Reiter, R. J., Zhang, H., et al. (2015b). Comparative physiological, metabolomic, and transcriptomic analyses reveal mechanisms of improved abiotic stress resistance in bermudagrass [Cynodon dactylon (L). Pers.] by exogenous melatonin. J. Exp. Bot. 66, 681-694. doi: 10.1093/jxb/eru373

Shi, H., Reiter, R. J., Tan, D. X., and Chan, Z. (2015c). INDOLE-3-ACETIC ACID INDUCIBLE 17 positively modulates natural leaf senescence through melatonin-mediated pathway in Arabidopsis. J. Pineal Res. 58, 26-33. doi: 10. 1111/jpi.12188

Shi, H., Tan, D. X., Reiter, R. J., Ye, T., Yang, F., and Chan, Z. (2015d). Melatonin induces class A1 heat shock factors (HSFA1s) and their possible involvement of thermotolerance in Arabidopsis. J. Pineal Res. 58, 335-342. doi: 10.1111/jpi. 12219

Shi, H., Wang, X., Tan, D. X., Reiter, R. J., and Chan, Z. (2015e). Comparative physiological and proteomic analyses reveal the actions of melatonin in the reduction of oxidative stress in bermudagrass (Cynodon dactylon (L). Pers.). J. Pineal Res. 59, 120-131. doi: 10.1111/jpi.12246

Shi, H., Wei, Y., Wang, Q., Reiter, R. J., and He, C. (2016). Melatonin mediates the stabilization of DELLA proteins to repress the floral transition in Arabidopsis. J. Pineal Res. 60, 373-379. doi: 10.1111/jpi.12320

Stepanova, A. N., Robertson-Hoyt, J., Yun, J., Benavente, L. M., Xie, D. Y., Doležal, K., et al. (2008). TAA1-mediated auxin biosynthesis is essential for hormone crosstalk and plant development. Cell 133, 177-191. doi: 10.1016/j. cell.2008.01.047

Strader, L. C., and Bartel, B. (2008). A new path to auxin. Nat. Chem. Biol. 4, 337-339. doi: 10.1038/nchembio0608-337

Strader, L. C., Wheeler, D. L., Christensen, S. E., Berens, J. C., Cohen, J. D., Rampey, R. A., et al. (2011). Multiple facets of Arabidopsis seedling development require indole-3-butyric acid-derived auxin. Plant Cell 23, 984-999. doi: 10.1105/tpc. 111.083071

Suzuki, M., Yamazaki, C., Mitsui, M., Kakei, Y., Mitani, Y., Nakamura, A., et al. (2015). Transcriptional feedback regulation of YUCCA genes in response to auxin levels in Arabidopsis. Plant Cell Rep. 34, 1343-1352. doi: 10.1007/s00299015-1791-Z

Tao, Y., Ferrer, J. L., Ljung, K., Pojer, F., Hong, F., Long, J. A., et al. (2008). Rapid synthesis of auxin via a new tryptophan-dependent pathway is required for shade avoidance in plants. Cell 133, 164-176. doi: 10.1016/j.cell.2008. 01.049

Teale, W. D., Paponov, I. A., Ditengou, F., and Palme, K. (2005). Auxin and the developing root of Arabidopsis thaliana. Physiol. Plant. 123, 130-138. doi: 10.1111/j.1399-3054.2005.00475.x
Ulmasov, T., Murfett, J., Hagen, G., and Guilfoyle, T. J. (1997). Aux/IAA proteins repress expression of reporter genes containing natural and highly active synthetic auxin response elements. Plant Cell 9, 1963-1971. doi: 10.1105/tpc. 9.11.1963

Verbelen, J. P., De Cnodder, T., Le, J., Vissenberg, K., and Baluska, F. (2006). The root apex of Arabidopsis thaliana consists of four distinct zones of growth activities: meristematic zone, transition zone, fast elongation zone and growth terminating zone. Plant Signal. Behav. 1, 296-304. doi: 10.4161/psb.1.6.3511

Wang, B., Chu, J., Yu, T., Xu, Q., Sun, X., Yuan, J., et al. (2015). Tryptophanindependent auxin biosynthesis contributes to early embryogenesis in Arabidopsis. PNAS 112, 4821-4826. doi: 10.1073/pnas.1503998112

Wang, P., Yin, L., Liang, D., Li, C., Ma, F., and Yue, Z. (2012). Delayed senescence of apple leaves by exogenous melatonin treatment: toward regulating the ascorbate-glutathione cycle. J. Pineal Res. 53, 11-20. doi: 10.1111/j.1600-079X. 2011.00966.x

Weeda, S., Zhang, N., Zhao, X., Ndip, G., Guo, Y., Buck, G. A., et al. (2014). Arabidopsis transcriptome analysis reveals key roles of melatonin in plant defense systems. PLoS ONE 9:e93462. doi: 10.1371/journal.pone.0093462

Yamamoto, Y., Kamiya, N., Morinaka, Y., Matsuoka, M., and Sazuka, T. (2007). Auxin biosynthesis by the YUCCA genes in rice. Plant Physiol. 143, 1362-1371. doi: 10.1104/pp.106.091561

Yin, L., Wang, P., Li, M., Ke, X., Li, C., Liang, D., et al. (2013). Exogenous melatonin improves Malus resistance to Marssonina apple blotch. J. Pineal Res. 54, 426-434. doi: 10.1111/jpi.12038

Yuan, H. M., and Huang, X. (2016). Inhibition of root meristem growth by cadmium involves nitric oxide-mediated repression of auxin accumulation and signalling in Arabidopsis. Plant Cell Environ. 39, 120-135. doi: 10.1111/pce. 12597

Zhang, N., Zhang, H. J., Zhao, B., Sun, Q. Q., Cao, Y. Y., Li, R., et al. (2014). The RNA-seq approach to discriminate gene expression profiles in response to melatonin on cucumber lateral root formation. J. Pineal Res. 56, 39-50. doi: $10.1111 /$ jpi.12095

Zhang, N., Zhao, B., Zhang, H. J., Weeda, S., Yang, C., Yang, Z. C., et al. (2013). Melatonin promotes water-stress tolerance, lateral root formation, and seed germination in cucumber (Cucumis sativus L.). J. Pineal Res. 54, 15-23. doi: 10.1111/j.1600-079X.2012.01015.x

Zhao, Y., Christensen, S. K., Fankhauser, C., Cashman, J. R., Cohen, J. D., Weigel, D., et al. (2001). A role for flavin monooxygenase-like enzymes in auxin biosynthesis. Science 291, 306-309. doi: 10.1126/science.291.5502.306

Zhao, Y., Tan, D. X., Lei, Q., Chen, H., Wang, L., Li, Q. T., et al. (2013). Melatonin and its potential biological functions in the fruits of sweet cherry. J. Pineal Res. 55, 79-88. doi: 10.1111/jpi.12044

Zuo, B., Zheng, X., He, P., Wang, L., Lei, Q., Feng, C., et al. (2014). Overexpression of MzASMT improves melatonin production and enhances drought tolerance in transgenic Arabidopsis thaliana plants. J. Pineal Res. 57, 408-417. doi: 10. $1111 /$ jpi.12180

Conflict of Interest Statement: The authors declare that the research was conducted in the absence of any commercial or financial relationships that could be construed as a potential conflict of interest.

Copyright (C) 2016 Wang, An, Wei, Reiter, Shi, Luo and He. This is an open-access article distributed under the terms of the Creative Commons Attribution License (CC BY). The use, distribution or reproduction in other forums is permitted, provided the original author(s) or licensor are credited and that the original publication in this journal is cited, in accordance with accepted academic practice. No use, distribution or reproduction is permitted which does not comply with these terms. 